\title{
Enhanced terminal room disinfection and acquisition and infection caused by multidrug-resistant organisms and Clostridium difficile (the Benefits of Enhanced Terminal Room Disinfection study): a cluster-randomised, multicentre, crossover study
}

\author{
DeverickJ Anderson, Luke F Chen, David J Weber, Rebekah W Moehring, Sarah S Lewis, Patricia F Triplett, Michael Blocker, Paul Becherer, \\ J Conrad Schwab, Lauren P Knelson, Yuliya Lokhnygina, William A Rutala, Hajime Kanamori, Maria F Gergen, Daniel J Sexton; for the CDC \\ Prevention Epicenters Program
}

\begin{abstract}
Summary
Background Patients admitted to hospital can acquire multidrug-resistant organisms and Clostridium difficile from inadequately disinfected environmental surfaces. We determined the effect of three enhanced strategies for terminal room disinfection (disinfection of a room between occupying patients) on acquisition and infection due to meticillinresistant Staphylococcus aureus, vancomycin-resistant enterococci, C difficile, and multidrug-resistant Acinetobacter.
\end{abstract}

Methods We did a pragmatic, cluster-randomised, crossover trial at nine hospitals in the southeastern USA. Rooms from which a patient with infection or colonisation with a target organism was discharged were terminally disinfected with one of four strategies: reference (quaternary ammonium disinfectant except for $C$ difficile, for which bleach was used); UV (quaternary ammonium disinfectant and disinfecting ultraviolet [UV-C] light except for C difficile, for which bleach and UV-C were used); bleach; and bleach and UV-C. The next patient admitted to the targeted room was considered exposed. Every strategy was used at each hospital in four consecutive 7-month periods. We randomly assigned the sequence of strategies for each hospital (1:1:1:1). The primary outcomes were the incidence of infection or colonisation with all target organisms among exposed patients and the incidence of $C$ difficile infection among exposed patients in the intention-to-treat population. This trial is registered with ClinicalTrials.gov, NCT01579370.

Findings 31226 patients were exposed; 21395 (69\%) met all inclusion criteria, including 4916 in the reference group, 5178 in the UV group, 5438 in the bleach group, and 5863 in the bleach and UV group. 115 patients had the primary outcome during 22426 exposure days in the reference group ( 51.3 per 10000 exposure days). The incidence of targe organisms among exposed patients was significantly lower after adding $U V$ to standard cleaning strategies $(n=76$; 33.9 cases per 10000 exposure days; relative risk [RR] $0 \cdot 70,95 \% \mathrm{CI} 0 \cdot 50-0 \cdot 98 ; \mathrm{p}=0.036$ ). The primary outcome was not statistically lower with bleach ( $\mathrm{n}=101 ; 41.6$ cases per 10000 exposure days; RR $0 \cdot 85,95 \% \mathrm{CI} 0 \cdot$. 69-1.04; $\mathrm{p}=0 \cdot 116)$, or bleach and $\mathrm{UV}(\mathrm{n}=131 ; 45 \cdot 6$ cases per 10000 exposure days; RR $0 \cdot 91,95 \% \mathrm{CI} 0 \cdot 76-1 \cdot 09 ; \mathrm{p}=0 \cdot 303$ ) among exposed patients. Similarly, the incidence of $C$ difficile infection among exposed patients was not changed after adding UV to cleaning with bleach $(\mathrm{n}=38$ vs $36 ; 30.4$ cases vs 31.6 cases per 10000 exposure days; RR $1 \cdot 0$, 95\% CI $0.57-1.75$; $\mathrm{p}=\mathbf{0}$.997).

Interpretation A contaminated health-care environment is an important source for acquisition of pathogens; enhanced terminal room disinfection decreases this risk.

Funding US Centers for Disease Control and Prevention.

\section{Introduction}

Multidrug-resistant organisms and Clostridium difficile are common causes of health-care-associated infections that lead to adverse patient outcomes. ${ }^{1}$ The hospital environment may be an important source for transmission of these organisms. First, hospitals are contaminated with clinically important multidrug-resistant organisms and $C$ difficile. ${ }^{2}$ Meticillin-resistant Staphylococcus aureus (MRSA), vancomycin-resistant enterococci (VRE), and Acinetobacter spp can survive on inanimate surfaces for days, and $C$ difficile can survive for months.
Second, only $50 \%$ of surfaces in hospital rooms are sufficiently cleaned between patient stays. ${ }^{4}$ As a result, patients admitted to rooms previously occupied by patients with multidrug-resistant organisms and $C$ difficile are at an increased risk of subsequent infection or colonisation with these organisms..$^{5}$ Finally, the contaminated environment is an important source of health-care personnel hand contamination..$^{6-8}$

Terminal room disinfection (disinfection of a room between occupying patients) can be enhanced by using a chemical disinfectant with sporicidal activity or by use of
Lancet 2017; 389: 805-14

Published Online January 16, 2017 http://dx.doi.org/10.1016/ S0140-6736(16)31588-4 See Comment page 765

Duke Infection Control Outreach Network, Division of Infectious Diseases (D) Anderson MD, LF Chen MBBS, SS Lewis MD LPKnelson MSPH, Prof D J Sexton MD), Department of Biostatistics and Bioinformatics (Y Lokhnygina PhD), Duke University Medical Center, Durham, NC, USA; Department of Hospital Epidemiology, University of North Carolina Health Care, Chapel Hill, NC, USA (Prof D J Weber MD PF Triplett MD, W A Rutala PhD $H$ Kanamori MD,

M F Gergen MT ASCP); Durham Veterans Affairs Medical Center, Durham, NC, USA (RW Moehring MD); High Point Regional Hospital, High Point, NC, USA (P F Triplett); Alamance Regional Medical Center, Burlington, NC, USA (M Blocker MD); Carolinas Medical Center, Charlotte, NC, USA (M Blocker); Rex Healthcare, Raleigh, NC, USA (P Becherer MD); and Chesapeake Regional Medical Center, Chesapeake, VA, USA (J C Schwab MD)

Correspondence to: Dr Deverick J Anderson, DUMC Box 102359, Durham, NC 27710, USA

deverick.anderson@duke.edu 
Research in context

\section{Evidence before this study}

The hospital environment is contaminated with multidrug-resistant organisms and Clostridium difficile and is often disinfected inadequately. As a result, patients who enter contaminated hospital rooms are at increased risk for acquisition and infection from these organisms. Enhanced disinfection strategies may decrease the risk for transmission of such bacteria through the hospital environment, but supportive evidence is limited to single centre or quasi-experimental studies. According to a systematic review by Han and colleagues, no randomised multicentre trials have been done to determine the efficacy of enhanced strategies.

\section{Added value of this study}

Our study is, to our knowledge, the first multicentre randomised controlled trial to evaluate the effect of enhanced

disinfection strategies on acquisition and infection due to four target organisms (meticillin-resistant Staphylococcus aureus, vancomycin-resistant staphylococci, multidrug-resistant Acinetobacter, and ( difficile). Adding a UV-C device to quaternary ammonium disinfection decreased the risk of subsequent acquisition and infection by target organisms. Our study shows the efficacy of enhanced disinfection and confirms that the contaminated hospital environment is a modifiable risk factor.

\section{Implications of all the available evidence}

Multidrug-resistant organisms and C difficile lead to adverse patient outcomes. Novel and improved prevention strategies are needed. Prevention of the spread of these organisms will probably require a multifaceted approach, including enhanced disinfection, improved hand hygiene, and antimicrobial stewardship. supplemental disinfection technologies. However, to our knowledge, no multicentre randomised assessment of enhanced terminal room disinfection strategies has been done. ${ }^{9}$ We designed the Benefits of Enhanced Terminal Room Disinfection study to assess the effects of four different strategies for terminal room disinfection on acquisition of multidrug-resistant organisms and $C$ difficile.

\section{Methods}

\section{Study design and participants}

We did this pragmatic, multicentre, cluster-randomised, crossover trial in nine hospitals in the USA from April, 2012, to July, 2014 (appendix). We tested one of four strategies for terminal room disinfection. Three strategies included enhanced terminal disinfection, and one included the standard terminal disinfection.

These four strategies were used in targeted rooms, defined as single-patient rooms from which a patient on contact precautions was discharged or transferred. In the reference group, targeted rooms were disinfected with quaternary ammonium-containing disinfectant for all rooms except those with patients with $C$ difficile, in which a hypochlorite-containing disinfectant (bleach) was used. In the UV group, targeted rooms were disinfected with quaternary ammonium-containing disinfectant and a UV-C device except rooms of patients with $C$ difficile, in which a bleach-containing disinfectant and UV-C device were used. In the bleach group, a bleach-containing disinfectant was used in all targeted rooms. In the bleach and UV group, a bleach-containing disinfectant and a UV-C device were used for all targeted rooms.

Each strategy was used at every study hospital for four consecutive 7-month study periods. Each study period consisted of a 1-month wash-in period followed by a 6-month period of data collection. The sequence of disinfection strategies was randomly selected for each hospital.

We selected study hospitals to include multiple types of hospitals (tertiary, community, Veterans Affairs) as a convenience sample. All microbiological cultures were considered for inclusion in our outcomes. Cultures may have been representative of infection or colonisation and included surveillance cultures, if obtained by policy at the study hospital. No screening cultures were obtained specifically for the study.

The Duke University Health System Institutional Review Board served as the central institutional review board. We received a waiver of informed consent for this study.

\section{Randomisation and masking}

We did resource-dependent randomisation of hospitals, taking into account the number of UV devices available (nine). First, we used a random number generator to determine the order in which hospitals would be randomly assigned a disinfection strategy. Then, we used a random number generator to determine the order in which disinfection strategies were used in each hospital. We continued this process for each hospital but counted the number of machines already assigned for other hospitals in each study period. If all nine UV-C devices were already assigned for a period, subsequent hospitals could not be assigned to one of the UV strategies for that period. Ultimately, all hospitals used all four strategies in a 1:1:1:1 ratio (appendix p 9). Allocation was not masked.

\section{Procedures}

All hospitals used gown and glove precautions (ie, contact precautions) for patients known or suspected to harbour multidrug-resistant organisms or $C$ difficile. Environmental services personnel were trained on the appropriate use of the disinfectants, cleaning 
protocols, and UV-C device. The appendix provides information on standardisation of disinfection practices, implementation, and measures of protocol fidelity (appendix pp 1-2).

We did a microbiological analysis of 92 randomly selected seed rooms at two study hospitals to determine the total and average number of colony-forming units of the four target organisms that remained in the hospital room after terminal room disinfection (appendix $\mathrm{p}$ 4). Microbiological analyses and identification were done with standard protocols. ${ }^{10}$ All hospitals used PCR-based nucleic acid amplification tests to identify $C$ difficile throughout the study.

We designed this study to detect infection or colonisation with one of four target organisms: MRSA, VRE, $C$ difficile, or multidrug-resistant Acinetobacter. ${ }^{11} \mathrm{~A}$ seed room was defined as a room containing a patient with microbiologically proven current or history of infection or colonisation with one or more target organisms. History of infection or colonisation was defined as any positive culture within the 12 months before admission. The next patient admitted to the seed room was an exposed patient. Community-onset was defined as the isolation of a target organism within the first $48 \mathrm{~h}$ of hospital admission. Hospital-acquired was defined as the isolation of a target organism after $48 \mathrm{~h}$ of hospital admission.

\section{Outcomes}

We had two primary outcomes: first, the incidence of all target organisms among patients exposed to seed rooms, and second, the incidence of $C$ difficile infection among patients exposed to seed rooms, in the intention-to-treat population. Secondary outcomes were incidence among exposed patients of MRSA, of VRE, and of multidrugresistant Acinetobacter; incidence in the whole hospital of all target organisms, of MRSA, of VRE, of $C$ difficile, and of multidrug-resistant Acinetobacter; and adverse events (rate of UV-C device failure, time on diversion, emergency room wait time, health-care worker perception of cleaning methods, and room turnover time [time between patient discharge and completion of terminal room disinfection]; appendix pp 4-5). Incidence was calculated as the number of qualifying incident cases per 10000 exposure days. Exposure days were calculated as the number of days the exposed patient spent in the seed room. Patients excluded from the numerator were also excluded from the denominator. Adverse outcomes were assessed at the hospital-level (ie, all patients or rooms were included in the analyses unless otherwise stated).

Three additional predetermined variables were measured at each study hospital: hand hygiene compliance, room cleaning compliance, and colonisation pressure (appendix pp 10-11). ${ }^{12}$ We obtained demographic data and comorbid conditions for all exposed patients

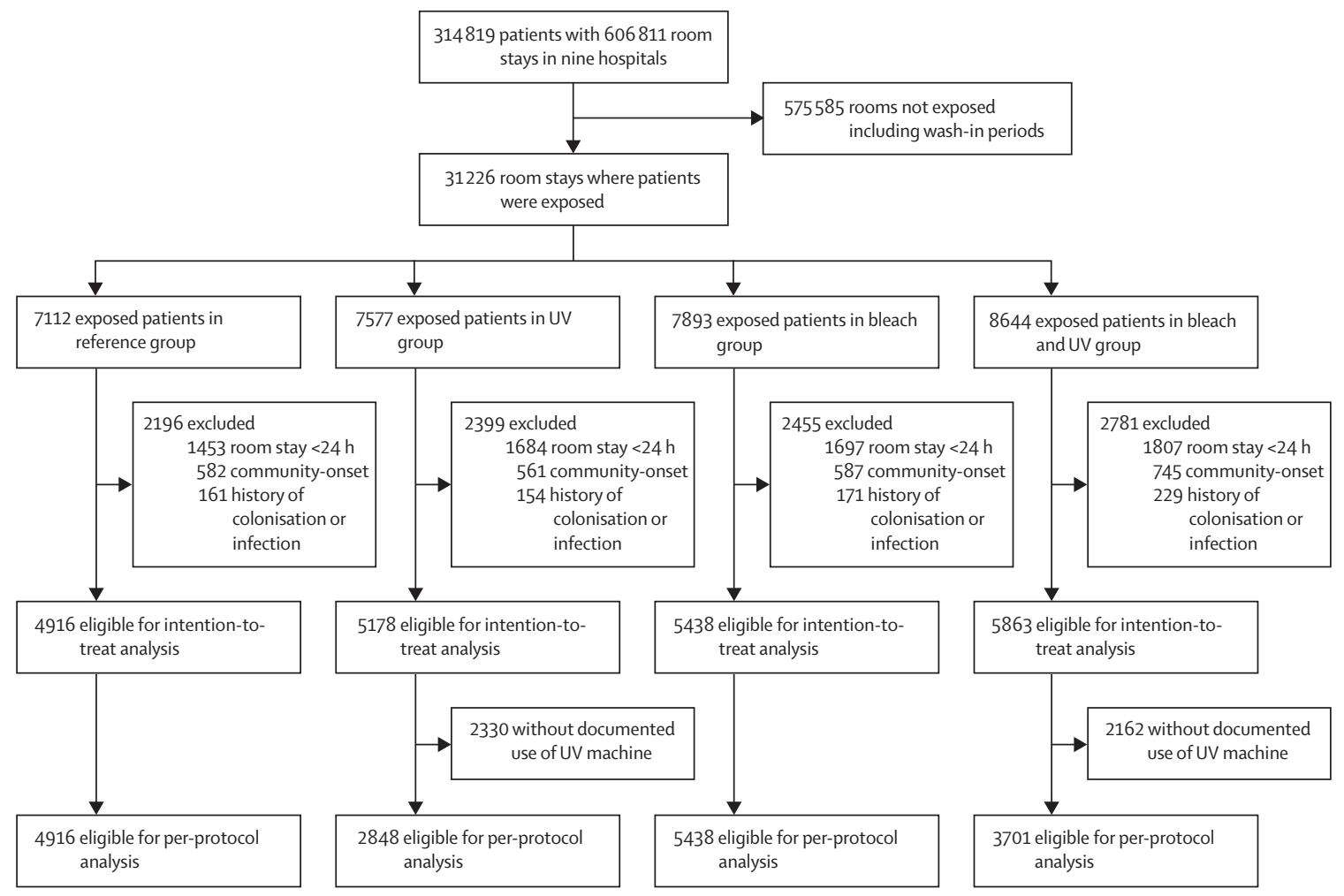

Figure: Trial profile 
through administrative databases to calculate Charlson scores. ${ }^{13}$

We did two post-hoc analyses: (1) of the incidence of target organisms among exposed patients after removing the criteria requiring a minimum of $24 \mathrm{~h}$ in the seed room; and (2) of the incidence of target organisms due to vegetative bacteria (MRSA, VRE, and multidrug-resistant Acinetobacter; appendix p 4).

Exposed patients qualified as an incident case of acquisition ${ }^{14}$ if they met the following criteria: in a seed room for $24 \mathrm{~h}$ or more AND a positive clinical culture or test with one of the target organisms AND the

\begin{tabular}{lcccc} 
& $\begin{array}{c}\text { Reference } \\
(\mathbf{n}=4916)\end{array}$ & $\begin{array}{c}\text { UV group } \\
(\mathbf{n}=5178)\end{array}$ & $\begin{array}{c}\text { Bleach group } \\
(\mathbf{n}=5438)\end{array}$ & $\begin{array}{c}\text { Bleach and UV } \\
(\mathbf{n}=5863)\end{array}$ \\
\hline Demographics* & & & & \\
Mean age (SD) & $57 \cdot 9(20 \cdot 9)$ & $58 \cdot 5(21 \cdot 3)$ & $58 \cdot 6(20 \cdot 7)$ & $57 \cdot 7(21 \cdot 8)$ \\
Race & & & & \\
$\quad$ White & $3042(63 \%)$ & $3228(65 \%)$ & $3416(64 \%)$ & $3747(64 \%)$ \\
African American & $1418(30 \%)$ & $1411(28 \%)$ & $1591(30 \%)$ & $1655(28 \%)$ \\
$\quad$ Other & $243(5 \%)$ & $233(5 \%)$ & $249(5 \%)$ & $329(6 \%)$ \\
$\quad$ Unknown & $102(2 \%)$ & $97(2 \%)$ & $95(2 \%)$ & $111(2 \%)$ \\
Male sex & $2475(51 \%)$ & $2518(51 \%)$ & $2768(52 \%)$ & $3017(52 \%)$ \\
Comorbidities* & & & & \\
Median Charlson index (IQR) & $2(1-4)$ & $2(0-4)$ & $2(1-4)$ & $2(0-4)$ \\
Myocardial infarction & $499(11 \%)$ & $457(10 \%)$ & $475(9 \%)$ & $583(10 \%)$ \\
Congestive heart failure & $937(20 \%)$ & $950(20 \%)$ & $1014(20 \%)$ & $1151(21 \%)$ \\
Cerebrovascular disease & $571(12 \%)$ & $540(11 \%)$ & $582(11 \%)$ & $610(11 \%)$ \\
Hemiplegia or paraplegia & $97(2 \%)$ & $118(2 \%)$ & $139(3 \%)$ & $166(3 \%)$ \\
Peripheral vascular disease & $450(10 \%)$ & $498(10 \%)$ & $524(10 \%)$ & $543(10 \%)$ \\
Dementia & $75(2 \%)$ & $101(2 \%)$ & $138(3 \%)$ & $111(2 \%)$ \\
COPD & $1248(27 \%)$ & $1325(28 \%)$ & $1339(26 \%)$ & $1516(27 \%)$ \\
Rheumatic disease & $161(3 \%)$ & $181(4 \%)$ & $183(4 \%)$ & $224(4 \%)$ \\
Peptic ulcer disease & $143(3 \%)$ & $97(2 \%)$ & $126(2 \%)$ & $178(3 \%)$ \\
Liver disease & & & & \\
$\quad$ Mild & $475(10 \%)$ & $452(9 \%)$ & $484(9 \%)$ & $557(10 \%)$ \\
$\quad$ Moderate or severe & $120(3 \%)$ & $142(3 \%)$ & $135(3 \%)$ & $177(3 \%)$ \\
Diabetes mellitus & $1302(28 \%)$ & $1248(26 \%)$ & $1371(27 \%)$ & $1505(27 \%)$ \\
$\quad$ Complicated & $303(7 \%)$ & $273(6 \%)$ & $350(7 \%)$ & $350(6 \%)$ \\
Renal disease & $980(21 \%)$ & $986(21 \%)$ & $1083(21 \%)$ & $1171(21 \%)$ \\
Malignancy & $842(18 \%)$ & $807(17 \%)$ & $864(17 \%)$ & $961(17 \%)$ \\
Metastatic solid tumour & $305(7 \%)$ & $340(7 \%)$ & $330(6 \%)$ & $367(7 \%)$ \\
AIDS/HIV & $49(1 \%)$ & $48(1 \%)$ & $52(1 \%)$ & $55(1 \%)$ \\
Arrhythmia & $1542(33 \%)$ & $1425(30 \%)$ & $1513(30 \%)$ & $1824(33 \%)$ \\
Valvular heart disease & $420(9 \%)$ & $415(9 \%)$ & $412(8 \%)$ & $566(10 \%)$ \\
Pulmonary circulation & $386(8 \%)$ & $452(9 \%)$ & $439(9 \%)$ & $495(9 \%)$ \\
disease & & & & \\
Hypertension & $526(18 \%)$ & $839(18 \%)$ & $959(19 \%)$ & $1007(18 \%)$ \\
$\quad$ Complicated & $665(14 \%)$ & $671(13 \%)$ & $792(14 \%)$ \\
Other neurological disease & $545(11 \%)$ & $599(12 \%)$ & $637(11 \%)$ \\
Hypothyroid disease & & & &
\end{tabular}

Data are n (\%) unless stated otherwise. *The majority of patient-specific data were not available from one study hospital because of changes in electronic health record systems as follows: age (406 had data missing), race (428 missing), sex (407 missing), comorbidity data (1267 missing). COPD=chronic obstructive pulmonary disease.

Table 1: Baseline characteristics organism identified in the clinical culture or test was the same target organism isolated from the preceding patient in the seed room AND the positive culture or test was obtained during the index admission either during exposure to the seed room OR the positive culture or test was obtained after exposure to the seed room during the index admission or readmission within 90 days of discharge from the room for MRSA VRE, and multidrug-resistant Acinetobacter ${ }^{15}$ or within 28 days of discharge from the room for $C$ difficile. ${ }^{16} \mathrm{We}$ excluded incident cases if they were community-onset infections or the exposed patient had a microbiologically proven history of infection or colonisation with the same target organism during the 12 months before admission.

\section{Statistical analysis}

We did power calculations based on a review of 4 years of surveillance data from study hospitals and published literature. All power calculations were done with twosided significance level of 0.05 . We projected that 1.96 million patient-days of care would be provided at the nine study hospitals (after excluding the wash-in periods). For each 6-month intervention period, we projected that approximately 491200 patient-days of care would occur (distributed across nine participating hospitals). Based on data from our pre-existing surveillance databases, we projected that 959 outcomes due to the four target organisms would occur during the baseline (or reference) 6-month period (ie, with standard terminal room disinfection and no use of UV-C), for a baseline incidence of 1.95 per 1000 patient-days. Under these assumptions, the study would have $60 \%$ power to detect a $10 \%$ decrease in incidence rate, $92 \%$ power to detect a $15 \%$ decrease, and more than $99 \%$ power to detect a $20 \%$ decrease. The power analysis was done using simulation and was based on a Poisson regression model with hospital-level incidence rate as the outcome and disinfection strategy and hospital as the covariates.

All qualifying incident cases were included in the intention-to-treat population. The per-protocol population was identical to the intention-to-treat population for the reference strategy and bleach strategy. For the two strategies involving the UV device, the per-protocol population included qualifying incident cases who entered a seed room with documented use of the UV device. For the purposes of this study, the UV device only had to be turned on, the cycle did not have to be completed. The appendix contains a more detailed discussion of the differences between the analysis populations (pp 2-3).

We summarised patient characteristics using percentages for categorical variables and medians for continuous variables. We analysed outcomes using intention-to-treat and per-protocol principles for outcomes among exposed patients. We analysed incidence rates using overdispersed Poisson models with 
disinfection group (reference, UV, bleach, and bleach and UV), order of the strategies within the study (whether a particular strategy was used in the first, second, third, or fourth study period), and hospital as fixed-effect categorical covariates. We used generalised estimating equations to account for correlation between different study strategies within the same hospital. Each of the study groups was compared to the reference group except for the analysis of $C$ difficile among exposed patients. Because this comparison involved the comparison of bleach vs bleach and UV-C, results from the UV group and the bleach and UV group were compared to results from the reference group and the bleach group. We used the same model construction strategy for all outcome analyses. We calculated relative risk (RR), 95\% CIs, and risk reductions for each model. Statistical tests were done at a two-sided significance level of $0 \cdot 05$. In light of the pragmatic nature of the trial, we made no adjustments for multiple comparisons. We did all statistical analyses using SAS (version 9.4).

The study is registered on ClinicalTrials.gov (NCT01579370).

\section{Role of the funding source}

The funder served an advisory role in the development of the study protocol. All authors had full access to all data in the study and had final responsibility for the decision to submit for publication.

\section{Results}

All nine hospitals participated in the study from April, 2012, to July, 2014. The appendix shows the

\begin{tabular}{|c|c|c|c|c|}
\hline & Reference & UV group & Bleach group & Bleach and UV group \\
\hline \multicolumn{5}{|l|}{ All target organisms } \\
\hline Exposed patients & 4916 & 5178 & 5438 & 5863 \\
\hline Incident cases (\%) & $115(2 \cdot 3 \%)$ & $76(1 \cdot 5 \%)$ & $101(1 \cdot 9 \%)$ & $131(2 \cdot 2 \%)$ \\
\hline Exposure days & 22426 & 22389 & 24261 & 28757 \\
\hline Rate (per 10000 exposure-days) & $51 \cdot 3$ & 33.9 & $41 \cdot 6$ & $45 \cdot 6$ \\
\hline Risk reduction $(95 \% \mathrm{Cl})$ & Reference & $17 \cdot 4$ (5.8 to $28 \cdot 9)$ & $9 \cdot 7(-2 \cdot 7$ to $22 \cdot 0)$ & $5 \cdot 7(-6 \cdot 2$ to $17 \cdot 7)$ \\
\hline RR $(95 \% \mathrm{Cl}) ; \mathrm{p}$ value & Reference & 0.70 (0.50 to 0.98$) ; 0.036$ & $0.85(0.69$ to 1.04$) ; 0.116$ & 0.91 (0.76 to 1.09$) ; 0.303$ \\
\hline \multicolumn{5}{|l|}{ Clostridium difficile* } \\
\hline Exposed patients & .. & .. & 2499 & 2678 \\
\hline Incident cases (\%) & .. & .. & $36(1 \cdot 4 \%)$ & $38(1 \cdot 4 \%)$ \\
\hline Exposure days & .. & .. & 11385 & 12509 \\
\hline Rate (per 10000 exposure-days) & .. & .. & 31.6 & $30 \cdot 4$ \\
\hline Risk reduction $(95 \% \mathrm{Cl})$ & .. & .. & Reference & $1 \cdot 2(-12 \cdot 7$ to $15 \cdot 2)$ \\
\hline RR (95\% Cl); p value & .. & .. & Reference & $1.0(0.57$ to 1.75$) ; 0.997$ \\
\hline \multicolumn{5}{|c|}{ Meticillin-resistant Staphylococcus aureus } \\
\hline Exposed patients & 3300 & 3451 & 3631 & 3848 \\
\hline Incident cases (\%) & $73(2 \cdot 2 \%)$ & $54(1 \cdot 6)$ & $74(2 \cdot 0)$ & $89(2 \cdot 3)$ \\
\hline Exposure days & 14524 & 14780 & 15343 & 18960 \\
\hline Rate (per 10000 exposure-days) & $50 \cdot 3$ & $36 \cdot 5$ & $48 \cdot 2$ & $46 \cdot 9$ \\
\hline Risk reduction $(95 \% \mathrm{Cl})$ & Reference & $13 \cdot 8(0 \cdot 1$ to $27 \cdot 3)$ & $2 \cdot 1(-13 \cdot 8$ to $17 \cdot 8)$ & $3.4(-8.9$ to $15 \cdot 5)$ \\
\hline RR $(95 \% \mathrm{Cl}) ; \mathrm{p}$ value & Reference & 0.78 (0.58 to 1.05$) ; 0.104$ & $1.00(0.82$ to 1.21$) ; 0.967$ & $0.97(0.78$ to 1.22$) ; 0.819$ \\
\hline \multicolumn{5}{|l|}{ Vancomycin-resistant enterococci } \\
\hline Exposed patients & 1055 & 1206 & 1468 & 1753 \\
\hline Incident cases (\%) & $37(3 \cdot 5 \%)$ & $17(1 \cdot 4 \%)$ & $24(1 \cdot 6 \%)$ & $37(2 \cdot 1 \%)$ \\
\hline Exposure days & 5838 & 5780 & 7522 & 9488 \\
\hline Rate (per 10000 exposure-days) & $63 \cdot 4$ & $29 \cdot 4$ & $31 \cdot 9$ & $39 \cdot 0$ \\
\hline Risk reduction $(95 \% \mathrm{Cl})$ & Reference & $34.0(9 \cdot 3$ to 58.6$)$ & $31 \cdot 5(12 \cdot 7$ to $50 \cdot 2)$ & $24 \cdot 4(0 \cdot 5$ to $48 \cdot 2)$ \\
\hline RR (95\% Cl); p-value & Reference & 0.41 (0.15 to 1.13$) ; 0.084$ & 0.43 (0.19 to 1.00$) ; 0.049$ & $0.36(0.18$ to 0.70$) ; 0.003$ \\
\hline \multicolumn{5}{|l|}{ Multidrug-resistant Acinetobacter $\dagger$} \\
\hline Exposed patients & 31 & 47 & 28 & 62 \\
\hline Incident cases (\%) & 0 & 0 & $1(3 \cdot 6)$ & 0 \\
\hline Exposure days & 156 & 199 & 98 & 244 \\
\hline Rate (per 10000 exposure-days) & 0 & 0 & $102 \cdot 4$ & 0 \\
\hline
\end{tabular}




\begin{tabular}{|c|c|c|c|c|}
\hline & Reference group & UV group & Bleach group & Bleach and UV group \\
\hline \multicolumn{5}{|l|}{ All target organisms } \\
\hline Exposed patients & 4916 & 2848 & 5438 & 3701 \\
\hline Incident cases (\%) & $115(2 \cdot 3 \%)$ & $46(1.6 \%)$ & $101(1 \cdot 9 \%)$ & $93(2 \cdot 5 \%)$ \\
\hline Exposure days & 22426 & 12299 & 24261 & 17354 \\
\hline Rate (per 10000 exposure-days) & $51 \cdot 3$ & $37 \cdot 4$ & $41 \cdot 6$ & $53 \cdot 6$ \\
\hline Risk reduction $(95 \% \mathrm{Cl})$ & Reference & $13 \cdot 9(-0.1$ to $27 \cdot 9)$ & $9 \cdot 7(-2 \cdot 7$ to $22 \cdot 0)$ & $-2 \cdot 3(-15 \cdot 7$ to $11 \cdot 1)$ \\
\hline RR $(95 \% \mathrm{Cl}) ; \mathrm{p}$ value & Reference & 0.69 (0.50 to 0.95$) ; 0.025$ & 0.74 (0.61 to 0.91$) ; 0.004$ & $1.0(0.81$ to 1.23$) ; 1.00$ \\
\hline \multicolumn{5}{|l|}{ Clostridium difficile ${ }^{*}$} \\
\hline Exposed patients & .. & .. & 2499 & 1712 \\
\hline Incident cases (\%) & .. &.. & $36(1.4 \%)$ & $30(1 \cdot 8 \%)$ \\
\hline Exposure days & .. & .. & 11385 & 8015 \\
\hline Rate (per 10000 exposure-days) & .. & .. & $31 \cdot 6$ & $37 \cdot 4$ \\
\hline Risk reduction $(95 \% \mathrm{Cl})$ &.. &.$\cdot$ & Reference & $-5 \cdot 8(-17 \cdot 1$ to $5 \cdot 5)$ \\
\hline RR (95\% Cl); p value & .. & .. & Reference & 1.22 (0.68 to 2.17$) ; 0.511$ \\
\hline \multicolumn{5}{|c|}{ Meticillin-resistant Staphylococcus aureus } \\
\hline Exposed patients & 3300 & 1872 & 3631 & 2425 \\
\hline Incident cases (\%) & $73(2 \cdot 2 \%)$ & $28(1 \cdot 5 \%)$ & $74(2 \cdot 0 \%)$ & $63(2 \cdot 6 \%)$ \\
\hline Exposure days & 14525 & 7934 & 15343 & 10681 \\
\hline Rate (per 10000 exposure-days) & $50 \cdot 3$ & $35 \cdot 3$ & $48 \cdot 2$ & $59 \cdot 0$ \\
\hline Risk reduction $(95 \% \mathrm{Cl})$ & Reference & $15 \cdot 0(-0 \cdot 6$ to $30 \cdot 6)$ & $2 \cdot 1(-13 \cdot 8$ to $17 \cdot 8)$ & $-8 \cdot 7(-18 \cdot 0$ to $0 \cdot 5)$ \\
\hline RR $(95 \% \mathrm{Cl}) ; \mathrm{p}$ value & Reference & 0.67 (0.48 to 0.94$) ; 0.019$ & 0.89 ( 0.72 to 1.09$) ; 0.260$ & 1.09 ( 0.85 to 1.39$) ; 0.503$ \\
\hline \multicolumn{5}{|l|}{ Vancomycin-resistant enterococci } \\
\hline Exposed patients & 1055 & 659 & 1468 & 1134 \\
\hline Incident cases (\%) & $37(3 \cdot 5 \%)$ & $13(2 \cdot 0 \%)$ & $24(1 \cdot 6 \%)$ & $24(2 \cdot 1 \%)$ \\
\hline Exposure days & 5838 & 3265 & 7522 & 6237 \\
\hline Rate (per 10000 exposure-days) & $63 \cdot 4$ & $39 \cdot 8$ & $31 \cdot 9$ & $38 \cdot 5$ \\
\hline Risk reduction $(95 \% \mathrm{Cl})$ & Reference & $23 \cdot 6(-6 \cdot 1$ to $53 \cdot 2)$ & $31 \cdot 5(12 \cdot 7$ to $50 \cdot 2)$ & $24.9(-0.6$ to 50.4$)$ \\
\hline RR $(95 \% \mathrm{Cl}) ; \mathrm{p}$ value & Reference & $0.56(0.21$ to 1.50$) ; 0.248$ & 0.35 (0.16 to 0.78$) ; 0.010$ & 0.41 ( 0.22 to 0.77$) ; 0.006$ \\
\hline
\end{tabular}

Data are unchanged for multidrug-resistant Acinetobacter baumaunii (table 2). ${ }^{*}$ Rooms with patients known or suspected of having $C$ difficile infection were terminally cleaned with hypochlorite-containing solutions.

Table 3: Results of per-protocol analysis

randomised assignment for each hospital (p 13). The average cluster size was 7807 (SD 644) patients. 31226 patients were exposed to a seed room; 24585 (79\%) stayed in the seed room for $24 \mathrm{~h}$ or more, and 21395 (69\%) met all inclusion criteria (figure). Baseline characteristics of qualifying exposed patients were similar for all four cleaning strategies (table 1).

A total of 423 outcomes were recorded: 228 (54\%) cultures represented infection and 195 (46\%) represented colonisation. 115 patients had a primary outcome during 22426 exposure days during the reference period (51.3 per 10000 exposure days); the median incidence of target organisms in the baseline period per hospital was $37 \cdot 1$ per 10000 exposure days (range 17.5-101.6). The addition of a UV-C device to the standard disinfection strategy significantly decreased the incidence of target organisms to 33.9 per 10000 exposure-days $(n=76$; RR $0 \cdot 70,95 \%$ CI $0 \cdot 50-0 \cdot 98 ; \mathrm{p}=0 \cdot 036$; table 2; appendix p 14). The incidence of target organisms was lower in eight of the nine study hospitals in the UV group (appendix p 15).

There was no significant difference in the incidence of target organisms from rooms treated with bleach compared with reference (table 2). Similarly, there was no significant difference between use of bleach and UV compared with reference (table 2). The appendix shows outcomes from individual study hospitals by intention to treat for each disinfection strategy (p 7).

The incidence of $C$ difficile was not significantly different with or without UV-C devices (table 2). The incidence of MRSA was not significantly lower in the UV group and essentially unchanged in the bleach and bleach and UV groups (table 2). The incidence of VRE was not significantly lower in the UV group but was significantly lower in both groups that used a bleach-containing disinfectant (table 2). The use of bleach decreased the incidence of VRE by $57 \%$ 
compared to reference; the use of bleach and a UV-C device decreased incidence of VRE by $64 \%$ (table 2). Only one patient acquired multidrug-resistant Acinetobacter after exposure in a seed room. Thus, no comparisons or models were constructed for this organism.

$2848(55 \%)$ of 5178 eligible rooms in the UV group and 3701 (63\%) of 5863 eligible of rooms in the bleach and UV group were included in the per-protocol analyses. Effect estimates were generally similar in per-protocol analyses to the intention-to-treat analyses (table 3). The incidence of MRSA, however, was signifi cantly lower in the UV group compared with the reference group (table 3).

Our microbiological assessment of 92 seed rooms after terminal disinfection showed that all enhanced strategies decreased the bioburden of target organisms, but the largest decrease occurred in the UV group (table 4). Protocol compliance, hand hygiene compliance, cleaning compliance, and colonisation pressure were similar across study groups (table 5; appendix p 4).

The median room cleaning time was approximately 4 min longer in the UV and UV and bleach groups (table 5). The total wait time in the emergency department and days on diversion were unchanged across disinfection strategies. Time from admit decision to departure from the emergency department was approximately 10-20 min longer in each of the enhanced disinfection groups compared with the reference group. One hospital reported a single UV-C exposure event during the study (appendix p 5). Additional secondary analyses, including incidence in the whole hospital of all target organisms, of MRSA, of VRE, of $C$ difficile, and of multidrug-resistant Acinetobacter, and health-care worker perception of cleaning methods, will be presented elsewhere.

We did two post-hoc analyses (appendix p 9). First, removing the 24-h exposure requirement for exposed patients did not change the eff ect measures. Second after excluding patients admitted to $C$ difficile seed rooms, the decrease in incidence of target vegetative multidrugresistant organisms was strengthened in the UV group and signifi cantly lower in the bleach and UV group.

\section{Discussion}

Our large, prospective, multicentre, cluster-randomised trial is the first, to our knowledge, to demonstrate a decrease in acquisition and infection with epidemiologically important pathogens following the use of enhanced room disinfection strategies. Patients admitted to rooms previously occupied by patients harbouring a multidrug-resistant organism or $C$ difficile were $10-30 \%$ less likely to acquire the same organism if the room was terminally disinfected using an enhanced strategy. The largest risk reduction occurred when a UV-C device was added to the standard disinfectant strategy. By contrast, we showed no statistically significant decrease in outcomes when we used enhanced terminal disinfection with bleach or bleach and UV. Similarly, the incidence of

\begin{tabular}{|c|c|c|c|c|c|c|c|c|}
\hline & \multicolumn{2}{|c|}{$\begin{array}{l}\text { Reference } \\
(\mathrm{n}=21)\end{array}$} & \multicolumn{2}{|c|}{$\begin{array}{l}\text { UV group } \\
(n=28)\end{array}$} & \multicolumn{2}{|c|}{$\begin{array}{l}\text { Bleach group } \\
(n=23)\end{array}$} & \multicolumn{2}{|c|}{$\begin{array}{l}\text { Bleach and UV } \\
\text { group }(n=20)\end{array}$} \\
\hline & $\begin{array}{l}\text { Total } \\
\text { CFU }\end{array}$ & $\begin{array}{l}\text { Mean CFU } \\
\text { per room } \\
(\mathrm{SD})\end{array}$ & $\begin{array}{l}\text { Total } \\
\text { CFU }\end{array}$ & $\begin{array}{l}\text { Mean CFU } \\
\text { per room } \\
(S D)\end{array}$ & $\begin{array}{l}\text { Total } \\
\text { CFU }\end{array}$ & $\begin{array}{l}\text { Mean CFU } \\
\text { per room } \\
(\mathrm{SD})\end{array}$ & $\begin{array}{l}\text { Total } \\
\text { CFU }\end{array}$ & $\begin{array}{l}\text { Mean CFU } \\
\text { per room } \\
(S D)\end{array}$ \\
\hline $\begin{array}{l}\text { Clostridium } \\
\text { difficile }\end{array}$ & 79 & $3 \cdot 8(14 \cdot 2)$ & 80 & $2 \cdot 9(12 \cdot 6)$ & 103 & $4 \cdot 5(14 \cdot 5)$ & 65 & $3 \cdot 3(10 \cdot 4)$ \\
\hline MRSA & 179 & $8 \cdot 5(27 \cdot 1)$ & 3 & $0.1(0.6)$ & 101 & $4 \cdot 4(15 \cdot 0)$ & 17 & $0.9(2.5)$ \\
\hline VRE & 831 & $39.6(127.5)$ & 6 & $0.2(0.8)$ & 56 & $2 \cdot 4(5 \cdot 6)$ & 38 & $1.9(6.1)$ \\
\hline $\begin{array}{l}\text { MDR } \\
\text { Acinetobacter spp }\end{array}$ & 188 & $9.0(36.4)$ & 5 & $0.2(0.9)$ & 9 & $0 \cdot 4(1.9)$ & 5 & $0.3(0.9)$ \\
\hline $\begin{array}{l}\text { Total target } \\
\text { organisms }\end{array}$ & 1277 & $60 \cdot 8(161 \cdot 3)$ & 94 & $3 \cdot 4(13 \cdot 4)$ & 269 & $11 \cdot 7(21 \cdot 4)$ & 125 & $6 \cdot 3(16 \cdot 1)$ \\
\hline
\end{tabular}

MRSA=meticillin-resistant Staphylococcus aureus. VRE=vancomycin-resistant enterococci. MDR=multidrug-resistant. $\mathrm{CFU}=$ colony-forming units.

Table 4: Microbiological assessment

$C$ difficile infection was not different among exposed patients after adding UV to bleach disinfection.

Our results need to be interpreted in the appropriate context. First, decreases in acquisition of target organisms associated with the use of enhanced disinfection strategies were recorded even though our reference group was also an enhanced strategy of sorts. Overall compliance with thoroughness of cleaning in the reference group was roughly $90 \%$. By contrast, most previous studies conclude that approximately half of all hospital room surfaces are not cleaned during terminal cleaning. ${ }^{4}$ Improved cleaning compliance decreases environmental bioburden ${ }^{17}$ and risk of acquisition, particularly of MRSA and VRE. ${ }^{18,19}$ Second, in the reference group, the quaternary ammonium-containing disinfectant was delivered with microfibre cloths, which remove more bacteria than cotton and synthetic fibre cloths. ${ }^{20}$ Third, the enhanced nature of the reference group and lack of multidrug-resistant Acinetobacter outcomes probably led to a decrease in power. Thus, the absence of a decrease in the incidence of target organisms among exposed patients in the bleach and bleach and UV groups might have been related to type II error.

No randomised controlled trials have previously been done using a UV device or enhanced chemical disinfectant. To our knowledge, only one other randomised controlled trial has investigated an enhanced terminal room disinfection strategy. A hydrogen peroxide vapour system was evaluated over 30 months in six high-risk units in a single tertiary care centre. ${ }^{21}$ Patients in intervention units had a $64 \%$ decrease in acquisition of multidrug-resistant organisms and $C$ difficile and a $75 \%$ decrease in acquisition of VRE compared to patients in control units.

UV devices reduce the environmental bioburden of MRSA, VRE, $C$ difficile, and Acinetobacter spp..$^{10,22}$ Of four published studies on the clinical effectiveness of UV devices, one showed a $20 \%$ decrease in hospital-acquired multidrug-resistant organisms ${ }^{23}$ and three showed $22-53 \%$ decreases in $C$ difficile infection. ${ }^{24-26}$ In light of 


\begin{tabular}{|c|c|c|c|c|}
\hline & Reference & UV group & Bleach group & Bleach and UV group \\
\hline \multicolumn{5}{|l|}{ Hospital-level variables } \\
\hline \multicolumn{5}{|l|}{ Hand hygiene compliance } \\
\hline Observations & 59519 & 64810 & 64950 & 57650 \\
\hline Median per hospital (IQR) & $89 \cdot 7(86 \cdot 3-94 \cdot 7)$ & $88 \cdot 1(84 \cdot 6-95 \cdot 7)$ & $91 \cdot 4(86 \cdot 2-96 \cdot 1)$ & $90 \cdot 8(82 \cdot 8-94 \cdot 1)$ \\
\hline \multicolumn{5}{|l|}{ Room cleaning } \\
\hline Room observations & 5717 & 4312 & 4538 & 5869 \\
\hline $\begin{array}{l}\text { Mean number of locations } \\
\text { monitored per room }\end{array}$ & $12 \cdot 4$ & $11 \cdot 6$ & $12 \cdot 5$ & $12 \cdot 0$ \\
\hline Locations monitored & 70704 & 50081 & 56753 & 70312 \\
\hline Median compliance (IQR) & $100 \%(91-100)$ & $95 \%(86-100)$ & $100 \%(87-100)$ & $100 \%(84-100)$ \\
\hline Median colonisation pressure (IQR) & $4 \cdot 6 \%(3 \cdot 1-9 \cdot 7)$ & $4 \cdot 3 \%(3 \cdot 6-6 \cdot 6)$ & $4 \cdot 5 \%(3 \cdot 7-5 \cdot 8)$ & $4 \cdot 8 \%(3 \cdot 4-6 \cdot 7)$ \\
\hline \multicolumn{5}{|l|}{ Protocol compliance } \\
\hline $\mathrm{pH}$ pen use $(\%)$ & $4836 / 5024(96 \%)$ & $2970 / 3262(91 \%)$ & $1161 / 1206(96 \%)$ & $5899 / 6002(98 \%)$ \\
\hline Median (IQR) & $100(91-100)$ & $93(89-97)$ & $96(95-98)$ & 99 (97-99) \\
\hline $\begin{array}{l}\text { UV-C devices used in contact } \\
\text { precaution rooms (\%) }\end{array}$ &. & $6214 / 7137(87 \%)$ & .. & $10006 / 11274(89 \%)$ \\
\hline Median (IQR) &.$\cdot$ & $92(85-93)$ &.$\cdot$ & $91(87-91)$ \\
\hline \multicolumn{5}{|l|}{ Adverse events } \\
\hline \multicolumn{5}{|l|}{ Room turnover times } \\
\hline Median total turnover time (IQR) & $79 \cdot 4(74 \cdot 4-117 \cdot 3)$ & $88 \cdot 9(80 \cdot 0-93 \cdot 4)$ & $82 \cdot 6(73 \cdot 1-123 \cdot 3)$ & $87 \cdot 5(76 \cdot 2-127 \cdot 0)$ \\
\hline Rooms & 78413 & 127028 & 114101 & 102227 \\
\hline Median room cleaning time (IQR) & $35 \cdot 9(32 \cdot 5-38 \cdot 5)$ & $40 \cdot 7(38 \cdot 4-42 \cdot 1)$ & $35 \cdot 6(32 \cdot 3-38 \cdot 9)$ & $40 \cdot 1(39 \cdot 1-44 \cdot 2)$ \\
\hline Rooms & 133744 & 144183 & 132753 & 137814 \\
\hline \multicolumn{5}{|l|}{$\begin{array}{l}\text { Emergency department waiting times } \\
\text { (min) }\end{array}$} \\
\hline $\begin{array}{l}\text { Median total time in emergency } \\
\text { department (range; } n=7 \text { ) }\end{array}$ & $392(290-537)$ & $390(286-534)$ & $392(290-533)$ & $399(294-544)$ \\
\hline Observations & 34532 & 31961 & 30613 & 32320 \\
\hline $\begin{array}{l}\text { Median time from admit decision } \\
\text { to departure from emergency } \\
\text { department (range; } n=4 \text { ) }\end{array}$ & $92(64-135)$ & $110(72-180)$ & $116(75-194)$ & $108(70-184)$ \\
\hline Observations & 18443 & 24025 & 21566 & 23732 \\
\hline \multicolumn{5}{|l|}{ Time on diversion (days) } \\
\hline Total & $63 \cdot 8$ & $53 \cdot 8$ & $34 \cdot 2$ & $38 \cdot 2$ \\
\hline Median per hospital (IQR) & $2 \cdot 7(0 \cdot 3-11 \cdot 7)$ & $2 \cdot 5(1 \cdot 7-7)$ & $2.9(0.6-7.5)$ & $1 \cdot 3(0 \cdot 6-6 \cdot 6)$ \\
\hline
\end{tabular}

these results, we were surprised by the lack of change in rates of $C$ difficile among exposed patients. This lack of change might have been caused by the following factors. First, the reference group for our $C$ difficile-specific outcome involved the use of bleach. As we had high (around 90\%) compliance with the use of bleach, there may have been relatively few residual spores for the UV device to eliminate. ${ }^{17}$ Second, UV is less effective against $C$ difficile than against vegetative bacteria, especially in areas of shadow. ${ }^{10,22}$ Third, we used a single-stage cycle with the UV-C device placed adjacent to but outside of the bathroom. ${ }^{27}$ Thus, we may not have effectively eliminated $C$ difficile from bathrooms. Finally, the environment might not play as large a role in $C$ difficile transmission as previously suspected. ${ }^{28}$ Eyre and colleagues ${ }^{29}$ assessed
1250 cases of symptomatic $C$ difficile in Oxfordshire, UK, over a 4year period using whole genome sequencing and reported that $45 \%$ of $C$ difficile cases were genetically distinct from previous cases. Although this analysis did not consider asymptomatic colonisation, only $2 \%$ of patients with related $C$ difficile isolates were linked by possible environmental contamination. Our post-hoc analysis excluding patients exposed to $C$ difficile showed that the effect in the UV group was strengthened and the effect in the bleach and UV group became statistically significant.

To our knowledge, no other randomised controlled trials have assessed the effect of using a sporicidal disinfectant on the incidence of our target organisms. Grabsch and colleagues ${ }^{30}$ recorded a $67 \%$ decrease in acquisition of VRE and an $83 \%$ decrease in VRE 
bacteraemia with use of bleach. Results from the intention-to-treat analysis in our trial did not show a signifi cant decrease in the incidence of target organisms following the routine use of bleach for terminal disinfection of contact precaution rooms. Results from a prespecifi ed secondary analysis, however, validate the decrease in VRE reported by Grabsch and colleagues. Our clinical results were largely corroborated by our microbiological assessment of seed rooms after terminal disinfection; greater reductions in colony-forming units occurred in the UV group than in the bleach and UV and bleach groups.

Our study has limitations. First, we relied on clinical cultures obtained during the course of standard care, which might have introduced ascertainment bias. Clinicians might have changed their culturing practices during the course of the study. Additionally, because we did not screen seed patients with a history of infection or colonisation or all exposed patients on exit from the seed rooms, we did not capture all acquisitions, might have failed to exclude a patient with community-onset colonisation, and our denominators might have included extra exposure days. We doubt, however, that any of these scenarios aff ected our results given randomisation. Second, we did not do molecular analyses to confirm that the organisms included in our outcomes were related to organisms in the environment, as this task was impossible given the scope of our study. Third, we did not account for multiplicity in our statistical testing given the pragmatic nature of our study; thus, the $\mathrm{p}$ values generated from our analyses should be interpreted with caution. Fourth, as noted above, our study had an enhanced reference group and thus decreased power. As a result, we suspect the eff ect measures in our study represent minimum effects of these strategies. Finally, our intervention was directed towards three multidrug-resistant organisms and $C$ difficile. We suspect that enhanced terminal room disinfection strategies decrease risk of acquisition of non-multidrugresistant organisms, such as meticillin-susceptible $S$ aureus and vancomycin-susceptible enterococci.

Acquisition and infection with multidrug-resistant organisms and $C$ difficile in health care is a complex and multifaceted process. Our study suggests that (1) the health-care environment is an important source for acquisition of multidrug-resistant organisms and $C$ difficile, and (2) the risk of acquisition of these pathogens from the environment can be modified. More than a century after Semmelweis and Lister's landmark studies, our results suggest that methods to improve disinfection can still lead to better patient outcomes.

\section{Contributors}

DJA did the literature search, prepared the figures, designed the study, collected, analysed, and interpreted data, wrote the Article, and approved the final version. LFC, DJW, RWM, SSL, WAR, and DJS designed the study, collected and interpreted data, revised the Article, and approved the final version. PFT, MB, PB, JCS, LPK, HK, and MGT collected data, revised the Article, and approved the final version. YL analysed and interpreted data, revised the Article, and approved the finalversion.

\section{Declaration of interests}

WAR and DIW have received consulting fees from Clorox. The other authors declare no competing interests.

\section{Acknowledgments}

Supported by grants from the CDC Prevention Epicenters Program (U54CK000164, to DJS), the NIH/NIAID (K23AI095357, to DJA), and the National Center for Advancing Translational Sciences, NIH (UL1TR001111). The views expressed in this Article are those of the authors and do not necessarily represent the views of the CDC or NIH. We thank Joyce Frederick for her significant contributions to this study. We also thank the following for their contributions to the successful completion of this study: Alamance Regional Medical Center-Mike Gover, Ron Halleen, Jean Keck, Melba Phillips, Clea Pulliam, Cameron Satterfield, Ashlei Williamson, Sara Wall; Chesapeake Regional Medical Center-Cynthia Boydston, Melanie Buski, Rollyn Cartwright, Joleen Connor, Monica Fuller, Larry Hires, Allison Johnson, Amie Prado, Arnell Pugh, Billy Richmond, Barbara Shields, Monita Short, Tiffany Silmon, Scavellas Slater, Mary Summerlin, Tammy Von Moll, Elizabeth Wade Duke Raleigh Hospital—Linda Alford, Donald Brown, Kim Catron, Connie Clark, Glenda Debord, Tammy Green, Neal Seigler, Kathryn Whitfield, Brittain Wood; Duke Regional HospitalKint Greenhouse, Mary Justesen, Cameron Satterfield, James Sessoms, Laura Smith, Vicki Tutor, Joy Vollers, James Walker; Duke University Hospital—Orlando Ackerson, Demario Harris, Pam Isaacs, Judith Megelich, Christopher O'Connor, Ornella Stewart, Christopher Vogelheim, Jessica Vondy; Durham Veterans Affairs Medical Center-Ben Blount, Joel Boggan, Orlando Brown, James Bunn, Dwayne Hicks, Sara Hoffman, William Hubbell, Steve McLeod, Mike Mejia, Larry Park, Jeffrey Robinson, Susan Wilkins, Roger Walsh, Christopher Woods; High Point Regional Hospital-Angelina Drews, Rocky Jameson, Judy Pemberton, Jennifer Pridgen, Cherrie Speagle, Barbara Tillman; Rex HealthcareSean Agard, Jeffrey Agricola, Joe Brown, Vicki Clark, Marty Cooney, Aku Fudzie, Zoe Gjertson, Debra Harris, Christopher Riley; University of North Carolina Health Care-Katie Crandall, Lula Daniels, Lauren DiBase, Sherie Goldbach, Kimberly Green, Kandi Herndon, Lori Osborne, Emily Pfaff, Orlando Reyes, Emily Sickbert-Bennett, Jeff Strickler, Jason Smith. We acknowledge TruD SmartUVC, Ecolab, and Clorox for their significant material contributions to the study. We also thank Angelica Corporation and Shared Linen Services, each for donating microfibre cloths to a study hospital. All of these companies provided materials for the completion of the study but played no role in the funding, design, analysis, or manuscript preparation.

\section{References}

1 Tackling drug-resistant infections globally: final report and recommendations. 2016. http://amr-review.org/sites/default/ files/160525_Final\%20paper_with\%20cover.pdf (accessed June 1, 2016).

2 Dancer SJ. Importance of the environment in meticillin-resistant Staphylococcus aureus acquisition: the case for hospital cleaning. Lancet Infect Dis 2008; 8: 101-13.

3 Kramer A, Schwebke I, Kampf G. How long do nosocomial pathogens persist on inanimate surfaces? A systematic review. BMC Infect Dis 2006; 6: 130.

4 Carling PC, Parry MM, Rupp ME, Po JL, Dick B, Von Beheren S. Improving cleaning of the environment surrounding patients in 36 acute care hospitals. Infect Control Hosp Epidemiol 2008; 29: 1035-41.

5 Mitchell BG, Dancer SJ, Anderson M, Dehn E. Risk of organism acquisition from prior room occupants: a systematic review and meta-analysis. J Hosp Infect 2015; 91: 211-27.

6 Guerrero DM, Nerandzic MM, Jury LA, Jinno S, Chang S, Donskey CJ. Acquisition of spores on gloved hands after contact with the skin of patients with Clostridium difficile infection and with environmental surfaces in their rooms. Am J Infect Control 2012 40: $556-58$.

7 Hayden MK, Blom DW, Lyle EA, Moore CG, Weinstein RA. Risk of hand or glove contamination after contact with patients colonized with vancomycin-resistant enterococcus or the colonized patients environment. Infect Control Hosp Epidemiol 2008; 29: 149-54. 
8 Stiefel U, Cadnum JL, Eckstein BC, Guerrero DM, Tima MA, Donskey CJ. Contamination of hands with methicillin-resistant Staphylococcus aureus after contact with environmental surfaces and after contact with the skin of colonized patients. Infect Control Hosp Epidemiol 2011; 32: 185-87.

9 Han JH, Sullivan N, Leas BF, Pegues DA, Kaczmarek JL, Unscheid CA. Cleaning hospital room surfaces to prevent health care-associated infections: a technical brief. Ann Intern Med 2015 163: 598-607.

10 Anderson DJ, Gergen MF, Smathers E, et al. Decontamination of targeted pathogens from patient rooms using an automated ultraviolet-C-emitting device. Infect Control Hosp Epidemiol 2013; 34: 466-71.

11 Magiorakos AP, Srinivasan A, Carey RB, et al. Multidrug-resistant, extensively drug-resistant and pandrug-resistant bacteria: an international expert proposal for interim standard definitions for acquired resistance. Clin Microbiol Infect 2012; 18: 268-81.

12 Bonten MJ, Slaughter S, Ambergen AW, et al. The role of "colonization pressure" in the spread of vancomycin-resistant enterococci: an important infection control variable. JAMA Intern Med 1998; 158: 1127-32.

13 Deyo RA, Cherkin DC, Ciol MA. Adapting a clinical comorbidity index for use with ICD-9-CM administrative databases. J Clin Epidemiol 1992; 45: 613-19.

14 Huang SS, Rifas-Shiman SL, Warren DK, et al. Improving methicillin-resistant Staphylococcus aureus surveillance and reporting in intensive care units. J Infect Dis 2007; 195: 330-38.

15 Avery TR, Kleinman KP, Klompas M, Aschengrau A, Huang SS Inclusion of 30-day postdischarge detection triples the incidence of hospital-onset methicillin-resistant Staphylococcus aureus. Infect Control Hosp Epidemiol 2012; 33: 114-21.

16 McDonald LC, Coignard B, Dubberke E, Song X, Horan T, Kutty PK. Recommendations for surveillance of Clostridium difficile-associated disease. Infect Control Hosp Epidemiol 2007; 28: 140-45.

17 Eckstein BC, Adams DA, Eckstein EC, et al. Reduction of Clostridium difficile and vancomycin-resistant Enterococcus contamination of environmental surfaces after an intervention to improve cleaning methods. BMC Infect Dis 2007; 7: 61.

18 Datta R, Platt R, Yokoe DS, Huang SS. Environmental cleaning intervention and risk of acquiring multidrug-resistant organism from prior room occupants. JAMA Intern Med 2011; 171: 491-94.

19 Hayden MK, Bonten MJ, Blom DW, Lyle EA, van de Vijver DA, Weinstein RA. Reduction in acquisition of vancomycin-resistant enterococcus after enforcement of routine environmental cleaning measures. Clin Infect Dis 2006; 42: 1552-60.
20 Smith DL, Gillanders S, Holah JT, Gush C. Assessing the efficacy of different microfibre cloths at removing surface micro-organisms associated with healthcare-associated infections. J Hosp Infect 2011; 78: 182-86.

21 Passaretti CL, Otter JA, Reich NG, et al. An evaluation of environmental decontamination with hydrogen peroxide vapor for reducing the risk of patient acquisition of multidrug-resistant organisms. Clin Infect Dis 2013; 56: 27-35.

22 Rutala WA, Gergen MF, Weber DJ. Room decontamination with UV radiation. Infect Control Hosp Epidemiol 2010; 31: 1025-29.

23 Haas JP, Menz J, Dusza S, Montecalvo MA. Implementation and impact of ultraviolet environmental disinfection in an acute care setting. Am J Infect Control 2014; 42: 586-90.

24 Levin J, Riley LS, Parrish C, English D, Ahn S. The effect of portable pulsed xenon ultraviolet light after terminal cleaning on hospital-associated Clostridium difficile infection in a community hospital. Am J Infect Control 2013; 41: 746-48.

25 Miller R, Simmons S, Dale C, Stachowiak J, Stibich M. Utilization and impact of a pulsed-xenon ultraviolet room disinfection system and multidisciplinary care team on Clostridium difficile in a longterm acute care facility. Am J Infect Control 2015; 43: 1350-53.

26 Nagaraja A, Visintainer P, Haas JP, Menz J, Wormser GP Montecalvo MA. Clostridium difficile infections before and during use of ultraviolet disinfection. Am J Infect Control 2015; 3: 940-45.

27 Boyce JM, Havill NL, Moore BA. Terminal decontamination of patient rooms using an automated mobile UV light unit. Infect Control Hosp Epidemiol 2011; 32: 737-42.

28 Walker AS, Eyre DW, Wyllie DH, et al. Characterisation of Clostridium difficile hospital ward-based transmission using extensive epidemiological data and molecular typing. PLoS Med 2012; 9: e1001172.

29 Eyre DW, Cule ML, Wilson DJ, et al. Diverse sources of C. difficile infection identified on whole-genome sequencing. $N$ Engl J Med 2013; 369: 1195-205.

30 Grabsch EA, Mahony AA, Cameron DR, et al. Significant reduction in vancomycin-resistant enterococcus colonization and bacteraemia after introduction of a bleach-based cleaning-disinfection programme. J Hosp Infect 2012; 82: 234-42. 